\title{
Stability of Parametric Intuitionistic Fuzzy Multi-Objective Fractional Transportation Problem
}

\author{
Mohamed A. El Sayed ${ }^{1,2, *}$, Mohamed A. El-Shorbagy $3,4\left(\mathbb{0}\right.$, Farahat A. Farahat $^{5}$, Aisha F. Fareed ${ }^{2}$ \\ and Mohamed A. Elsisy 2,6 \\ 1 Basic Sciences Department, Faculty of Engineering, Badr University in Cairo BUC, Cairo 11829, Egypt \\ 2 Department of Basic Engineering Sciences, Faculty of Engineering, Benha University, Benha 13511, Egypt; \\ Aisha.farid@yahoo.com (A.F.F.); elsisy.mohamed@yahoo.com (M.A.E.) \\ 3 Department of Mathematics, College of Science and Humanities in Al-Kharj, Prince Sattam bin Abdulaziz \\ University, Al-Kharj 11942, Saudi Arabia; mohammed_shorbagy@yahoo.com or ma.hassan@psau.edu.sa \\ 4 Department of Basic Engineering Science, Faculty of Engineering, Menoufia University, \\ Shebin El-Kom 32511, Egypt \\ 5 Higher Technological Institute, Tenth of Ramadan City 44637, Egypt; Farahat_math@yahoo.com \\ 6 Department of Basic Engineering Sciences, Menoufia Higher Institute of Engineering and Technologey, \\ El-Bagour 32821, Egypt \\ * Correspondence: Mohamed.ali@bhit.bu.edu.eg or mohamedali20008@yahoo.com
}

Citation: El Sayed, M.A.;

El-Shorbagy, M.A.; Farahat, F.A.;

Fareed, A.F.; Elsisy, M.A. Stability of Parametric Intuitionistic Fuzzy Multi-Objective Fractional Transportation Problem. Fractal Fract. 2021, 5, 233. https://doi.org/ $10.3390 /$ fractalfract 5040233

Academic Editor: Savin Treanţă

Received: 14 October 2021

Accepted: 8 November 2021

Published: 19 November 2021

Publisher's Note: MDPI stays neutral with regard to jurisdictional claims in published maps and institutional affiliations.

Copyright: (c) 2021 by the authors. Licensee MDPI, Basel, Switzerland. This article is an open access article distributed under the terms and conditions of the Creative Commons Attribution (CC BY) license (https:/ / creativecommons.org/licenses/by/ $4.0 /)$.

\begin{abstract}
In this study, a parametric intuitionistic fuzzy multi-objective fractional transportation problem (PIF-MOFTP) is proposed. The current PIF-MOFTP has a single-scalar parameter in the objective functions and an intuitionistic fuzzy supply and demand. Based on the $(\alpha, \beta)$-cut concept a parametric $(\alpha, \beta)$-MOFTP is established. Then, a fuzzy goal programming (FGP) approach is utilized to obtain $(\alpha, \beta)$-Pareto optimal solution. We investigated the stability set of the first kind (SSFK) corresponding to the solution by extending the Kuhn-Tucker optimality conditions of multi-objective programming problems. An algorithm to crystalize the progressing SSFK for PIF-MOFTP as well as an illustrative numerical example is presented.
\end{abstract}

Keywords: multi-objective programming; fractional transportation problem; intuitionistic fuzzy set; parametric programming

\section{Introduction}

Transportation issues (TP) have been studied in various writings [1-7]. These issues and their solution processes postulate a worthy task in logistics and supply chain organization for reducing expenses, further developing service quality, etc. [3,8]. Nonetheless, $\mathrm{TP}$ is described by multiple, incommensurable, and clashing objective functions, being known as the multi-objective transportation problem (MO-TP). Accordingly, in MO-TP, the idea of an ideal solution offers spot to the idea of the best compromise solution or the non-dominated solutions. Optimization of the ratio of two functions is called fractional programming (ratio optimization) [7,9]. To be sure, in such circumstances, it is often a question of optimizing a ratio of benefit/cost, stock/deals, specialist/patient, and so on, subject to some constraints $[7,9]$.

One of the significant issues looked at by specialists is that involving the exact values of parameters [7]. In this way, this might involve thinking about vagueness, or specifying the fundamental parameters of the model, which are the coefficients of the objective function and the constrains [4,8]. Accordingly, it might be naturalistic to take the distinct adjectival information on specialists and leaders about the parameters which can be exemplified as fuzzy data $[7,10]$. Uncertainty may happen because of the accompanying unrestrained factors. In this study the main hypotheses are that the transportation charge has a parametric nature, and the supply and the demand parameters are intuitionistic 
fuzzy numbers (IFNs). The main hypotheses have not been presented in the literature, and the basic question is how we can get the SSFK for such PIF-MOFTP.

\section{Literature Review}

The research on MO-TP is improved by fusing the diverse numerical models and procedures. James et al. [11] examined transportation administration quality dependent on data combination. A lot of examination that deals with transportation wellbeing was created by Ergun et al. [12], Sheu and Chen [13]. Recently, MO-TP under different circumstances has been discussed by Roy et al. [14,15], Roy and Mahapatra [16], Roy [17], Maity and Roy $[18,19]$.

Although fuzzy set theory (FST) is novel tool in handling uncertainties, it cannot tackle special kinds of uncertainties, as it is difficult to depict the membership degree using one specific value. To overcome the lack of knowledge of non-membership degrees, intuitionistic fuzzy set (IFS) was presented in 1986 by Atanassov [20] as an extension of FST. In IFS, each element in a set is attached with two grades: membership and non-membership, where the sum of these two grades is restricted to less or equal to one. Moreover, many creators have been utilized IFS for addressing various sorts of TPs [21,22]. The study of MO-TP with vague numbers has been presented by Ammar and Youness [1]. The fuzzy programming strategy was acquainted with tackle MO-TP with various non-linear membership functions [23]. IFS has additionally been utilized by several scientists to tackle different types of TPs $[10,24]$. One more strategy for thoroughly considering linear MOTPs with vague nature has been suggested by Gupta and Kumar [25]. Recently, MO-TP under various types of uncertainty has been discussed by Roy and Mahapatra [16], Maity and Roy [26], and Ebrahimnejad and Verdegay [10]. Mahajan and Gupta [27] proposed a fully IF MO-TP utilizing various membership functions. Achievement stability set for parametric linear FGP problems has been introduced by El Sayed and Farahat [28]. The neutrosophic goal programming approach for solving the multi-objective fractional transportation problem was introduced by Veeramani et al., [29]. Pramanik and Banerjee [30] proposed a chance-constrained capacitated MO-TP with two fuzzy goals, and a consensus solution was found. Edalatpanah [31] developed a nonlinear framework for neutrosophic linear programming. Furthermore, Rizk-Allah et al. [32] developed a compromise solution framework for the MO-TP based on the neutrosophic environment. A fuzzy approach using generalized dinkelbach's algorithm for linear multi-objective fractional transportation problem (MOFTP) has been presented by Cetin and Tiryaki [3]. A fuzzy mathematical programming approach for solving fuzzy linear fractional programming problem has been demonstrated by Veeramani and Sumathi [33]. El Sayed and Abo-Sinna [7] introduced the intuitionistic fuzzy multi-objective fractional transportation problem (IF-MOFTP).

Parametric programming examines the impact of preordained continuous varieties in the objective function coefficients and the right-hand side of the constraints on the ideal solution [34-36]. In parametric analysis the objective function and the right-hand side vectors are replaced with the parameterized function $\boldsymbol{c}(\vartheta)$ and $\boldsymbol{b}(\alpha, \beta)$, where $\vartheta$ and $\alpha, \beta$ are the parameter of variation. The general idea of parametric analysis is to start with the $\alpha$-Pareto optimal solution at $\vartheta=\vartheta^{*}, \alpha=\alpha^{*}, \beta=\beta^{*}$. Then by applying KKT optimality the SSFK is determined [35,37]. The concept of the stability set of the first kind (SSFK) has been introduced by Osman [35], and extended by Saad [38], Saad and Hughes [39], Osman et al. [36], Saad et al. [40].

In prior examinations, the MO-TP was created with the presumption that the supply, demand, and cost boundaries were known. Nonetheless, applications, every one of the parameters of the TP are not for the most part characterized definitively. It might have IF values. Comparable contemplations might be taken for supply and demand parameters in TP of this paper. Keeping this perspective, the primary commitments are concerned with two unique viewpoints: one is to find a $(\alpha, \beta)$-Pareto optimal solution for the PIFMOFTP, and another is to investigate the SSFK for PIF-MOFTP. First, based on the $(\alpha, \beta)$-cut methodology a parametric $(\alpha, \beta)$-MOFTP is established. Then, A FGP approach is used to 
get $(\alpha, \beta)$-Pareto optimal solution. Finally, the KKT optimality conditions applied to get the SSFK. An algorithm to clarify the developed SSFK for PIF-MOFTP as well as an illustrative numerical example are given.

The rest of this study is organized as follows: after the introduction and literature review, Section 3 introduces some basic concepts. Modelling of the PIF-MOFTP is presented in Section 4. Section 5 demonstrates the FGP methodology for tackling the PIF-MOFTP. In the next section the SSFK is investigated. An algorithm for obtaining the SSFK for PIF-MOFTP is introduced in Section 6. An illustrative example, discussion and limitations is given in Section 7. This paper ends with some concluding remarks.

\section{Preliminaries}

This part presents the concept of IFS [20,21,41,42].

Definition 1. An IFS $\widetilde{A}^{I}$ in $X$ is a set of ordered triples $\widetilde{A}^{I}=\left\{\left(x, \mu_{\widetilde{A}^{I}}(x), v_{\widetilde{A}^{I}}(x)\right) \mid x \in X\right\}$, where $\mu_{\widetilde{A}^{I}}(x), v_{\widetilde{A}^{I}}(x): X \rightarrow[0,1]$ are functions such that $0 \leq \mu_{\widetilde{A}^{I}}(x)+v_{\widetilde{A}^{I}}(x) \leq 1, \forall x \in X$. The value of $\mu_{\widetilde{A}^{I}}(x)$ acts as the grade of membership and $v_{\widetilde{A}^{I}}(x)$ acts as the grade of non-membership of the element $x \in X$ being in $\widetilde{A}^{I} . h(x)=1-\mu_{\widetilde{A}^{I}}(x)-v_{\widetilde{A}^{I}}(x)$ represents the grade of hesitation for the element $x$ in $\widetilde{A}^{I}[20,41]$.

Definition 2. An IFN of the form $\widetilde{A}^{I}=\left(a_{1}, a_{2}, a_{3} ; \bar{a}_{1}, a_{2}, \bar{a}_{3}\right)$ is said to be triangular IFN (TIFN) with membership and non-membership functions defined as [41,43]:

$$
\begin{gathered}
\mu_{\widetilde{A}^{I}}(x)=\left\{\begin{array}{cc}
\frac{x-a_{1}}{a_{2}-a_{1}}, & a_{1} \leq x \leq a_{2}, \\
\frac{a_{3}-x}{a_{3}-a_{2}}, & a_{2} \leq x \leq a_{3} \\
0, & \text { otherwise }
\end{array}\right. \\
v_{\widetilde{A}^{I}}(x)=\left\{\begin{array}{cc}
\frac{a_{2}-x}{a_{2}-\bar{a}_{1}} & \bar{a}_{1} \leq x \leq a_{2} \\
\frac{x-a_{2}}{\bar{a}_{3}-a_{2}} & a_{2} \leq x \leq \bar{a}_{3} \\
1 & \text { otherwise }
\end{array}\right.
\end{gathered}
$$

where $\frac{x-a_{1}}{a_{2}-a_{1}}$, and $\frac{x-a_{2}}{\bar{a}_{3}-a_{2}}$ are continuous monotone increasing functions, $\frac{a_{3}-x}{a_{3}-a_{2}}$ and $\frac{a_{2}-x}{a_{2}-\bar{a}_{1}}$ are continuous monotone decreasing functions. $\frac{x-a_{1}}{a_{2}-a_{1}}, \frac{a_{3}-x}{a_{3}-a_{2}}, \frac{a_{2}-x}{a_{2}-\bar{a}_{1}}$ and $\frac{x-a_{2}}{\bar{a}_{3}-a_{2}}$ are the left and the right basis functions of the membership function and the non-membership function (see Figure 1), respectively. $\bar{a}_{1} \leq a_{1} \leq a_{2} \leq a_{3} \leq \bar{a}_{3}$ and $0 \leq \mu_{\widetilde{A}^{I}}(x)+v_{\widetilde{A}^{I}}(x) \leq 1, \forall x \in X$.

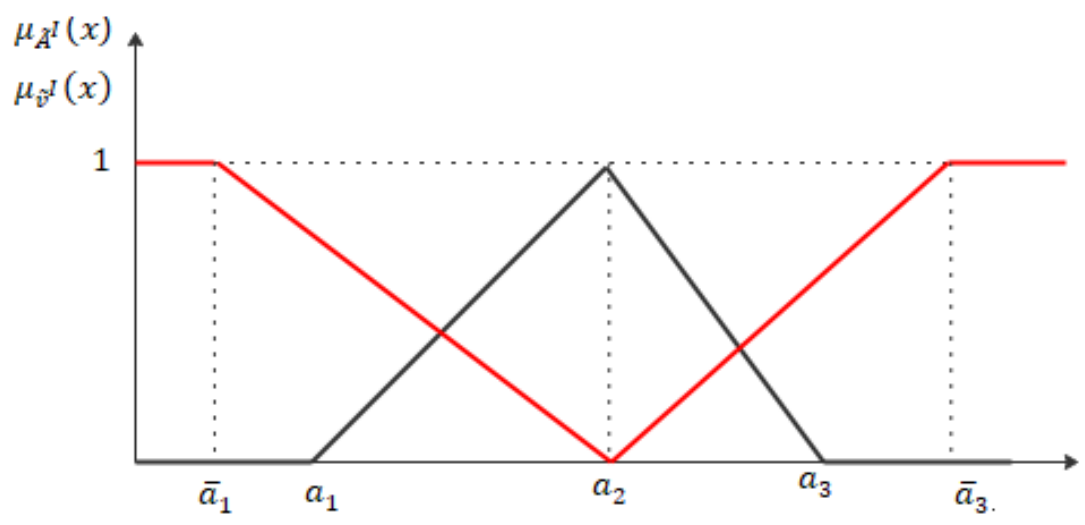

Figure 1. Triangular Intuitionistic Fuzzy number.

Definition 3. A TIFNs $\widetilde{A}^{I}=\left(a_{1}, a_{2}, a_{3} ; \bar{a}_{1}, a_{2}, \bar{a}_{3}\right)$ is assumed to be a non-negative TIFN iff, $\bar{a}_{1} \geq 0[41,43]$. 
Definition 4. Two TIFNs $\widetilde{A}^{I}=\left(a_{1}, a_{2}, a_{3} ; \bar{a}_{1}, a_{2}, \bar{a}_{3}\right)$ and $\widetilde{A}^{I}=\left(b_{1}, b_{2}, b_{3} ; \bar{b}_{1}, b_{2}, \bar{b}_{3}\right)$ are equivalent to one another, $\widetilde{A}^{I}=\widetilde{B}^{I}$ iff, $a_{i}=b_{i}$ and $\bar{a}_{i}=\bar{b}_{i} \forall i=1,2,3[7,41,43]$.

Definition 5. $(\alpha, \beta)$-cut of an IFS $\widetilde{A}^{I}$ is defined by: $\widetilde{A}_{(\alpha, \beta)}^{I}=\left\{x: \mu_{\widetilde{A}^{I}}(x) \geq \alpha, v_{\widetilde{A}^{I}}(x) \leq \beta\right.$, $\alpha+\beta \leq 1, x \in X\}$; where $\alpha, \beta \in(0,1]$.

Definition 6. $(\alpha, \beta)$-cut of a TIFN $\widetilde{A}^{I}=\left(a_{1}, a_{2}, a_{3} ; \bar{a}_{1}, a_{2}, \bar{a}_{3}\right)$ is the set of all $x$ whose degree of membership is greater than or equal to $\alpha$ and degree of non-membership is less than or equal to $\beta$, i.e., $\widetilde{A}_{(\alpha, \beta)}^{I}=\left\{x: \mu_{\widetilde{A}^{I}}(x) \geq \alpha, v_{\widetilde{A}^{I}}(x) \leq \beta, \alpha+\beta \leq 1, x \in X\right\}$.

The $(\alpha, \beta)$-cut of a TIFN is shown in Figure 2, is defined as the crisp set of elements $x$ which belong to $\widetilde{A}^{I}$ at least to the degree $\alpha$ and which does belong to $\widetilde{A}^{I}$ at most to the degree $\beta$.

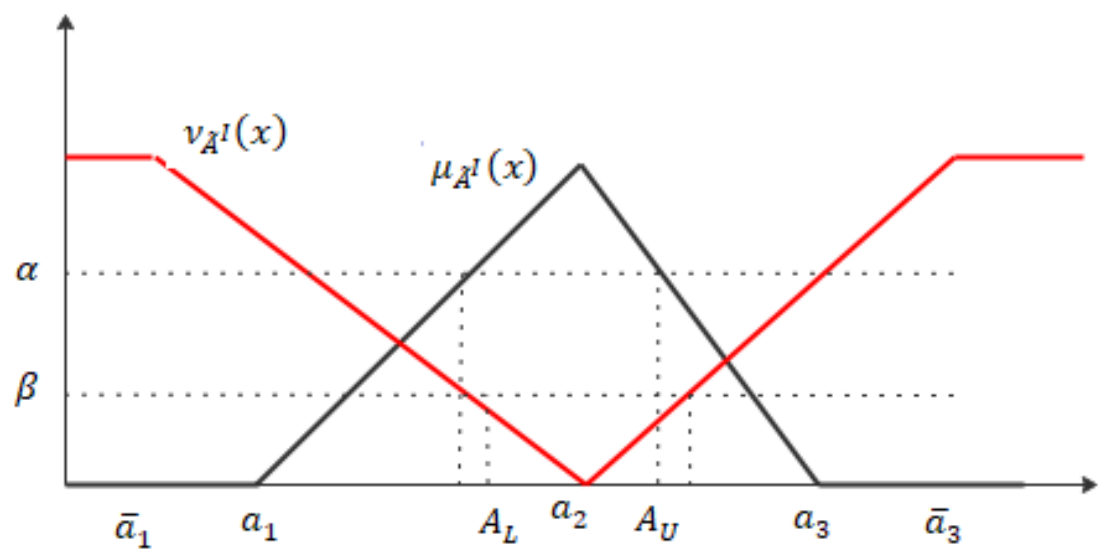

Figure 2. The $(\alpha, \beta)$-cut of a TIFN.

Now, $\mu_{\widetilde{A}^{I}}(x) \geq \alpha \Rightarrow \frac{x-a_{1}}{a_{2}-a_{1}} \geq \alpha$ and $\frac{a_{3}-x}{a_{3}-a_{2}} \geq \alpha$, or $x \geq a_{1}+\alpha\left(a_{2}-a_{1}\right)$ and $x \leq a_{3}-$ $\alpha\left(a_{3}-a_{2}\right)$ again, $v_{\widetilde{A}^{I}}(x) \leq \beta \Rightarrow \frac{a_{2}-x}{a_{2}-\bar{a}_{1}} \leq \beta$ and $\frac{x-a_{2}}{\bar{a}_{3}-a_{2}} \leq \beta$, or $x \geq a_{2}-\beta\left(a_{2}-\bar{a}_{1}\right)$ and $x \leq a_{2}+\beta\left(\bar{a}_{3}-a_{2}\right)$ [43]. Thus, referring to Figure $2 \widetilde{A}_{(\alpha, \beta)}^{I}=\left[A_{L}, A_{U}\right]$, where $A_{L}=$ $\max \left\{a_{1}+\alpha\left(a_{2}-a_{1}\right), a_{2}-\beta\left(a_{2}-\bar{a}_{1}\right)\right\}$ and $A_{U}=\min \left\{a_{3}-\alpha\left(a_{3}-a_{2}\right), a_{2}+\beta\left(\bar{a}_{3}-a_{2}\right)\right\}$.

\section{Mathematical Formulation}

In genuine case $\mathrm{TP}$, during the modeling process, the transportation parameters are not precise on account of insufficient information the variance of the market situation. To deal quantitatively with such unclear information, we deemed parametric IF-MOFTP in which single-scalar parameter $\vartheta \in \mathbb{R}$ in the objective functions and an intuitionistic fuzzy supply and demand. Suppose that there are $m$ sources and $n$ destinations. Thus, modelling of the parametric IF-MOFTP can be obtained as [3,7,9]:

$$
\operatorname{Max} Z_{q}(x, \vartheta)=\frac{\sum_{i=1}^{m} \sum_{j=1}^{n}\left(c_{i j}+\vartheta \omega_{i j}\right)^{(q)} x_{i j}^{(q)}+\delta^{(q)}}{\sum_{i=1}^{m} \sum_{j=1}^{n} d_{i j}^{(q)} x_{i j}^{(q)}+\rho^{(q)}}, q=1,2, \ldots, Q,
$$

\section{Subject to:}

$$
\begin{gathered}
\sum_{j=1}^{n} x_{i j} \leq \widetilde{a}_{i}^{I}, \quad i=1,2, \ldots, m, \\
\sum_{i=1}^{m} x_{i j} \geq \widetilde{b}_{j}^{I}, \quad j=1,2, \ldots, n,
\end{gathered}
$$




$$
x_{i j} \geq 0, \quad i=1,2, \ldots, m, j=1,2, \ldots, n .
$$

where $c_{i j}^{(q)}=\left(c_{i j}+\vartheta \omega_{i j}\right)^{(q)}$ denotes the parametric profit gained from shipment of $i^{\text {th }}$ source to $j^{\text {th }}$ destination. Also, $d_{i j}^{(q)}$ denotes the expense per unit of shipment from $i^{\text {th }}$ source to $j^{\text {th }}$ destination. $\delta^{(q)}, \rho^{(q)}$ are some constant profit and cost, respectively. $x_{i j}^{(q)}$ is the quantity shipped from $i^{t h}$ source to $j^{\text {th }}$ destination. $\widetilde{a}_{i}^{I}=\left(a_{i}^{1}, a_{i}^{2}, a_{i}^{3} ; \bar{a}_{i}^{1}, a_{i}^{2}, \bar{a}_{i}^{3}\right)$ stands for the available intuitionistic fuzzy supply at $i^{\text {th }}$ source and $\widetilde{b}_{j}^{I}=\left(b_{j}^{1}, b_{j}^{2}, b_{j}^{3} ; \bar{b}_{j}^{1}, b_{j}^{2}, \bar{b}_{j}^{3}\right)$ alludes to the accessible intuitionistic fuzzy demand at $j^{\text {th }}$ destination. Further, we postulate that $\sum_{i=1}^{m} \sum_{j=1}^{n} d_{i j}^{(q)} x_{i j}^{(q)}+\rho^{(q)}>0, q=1,2, \ldots, Q ; \widetilde{a}_{i}^{I}>0^{I}, \widetilde{b}_{j}^{I}>0^{I}, \forall j ;\left(c_{i j}+\vartheta \omega_{i j}\right)^{(q)}>$ $0^{I}, \delta^{(q)}, \rho^{(q)}>0$ for all $i, j$, and the gross supply is greater than or equal the gross demand $[3,7]$.

$$
\sum_{i=1}^{m}\left(\widetilde{a}_{i}^{I}\right)_{(\alpha, \beta)} \geq \sum_{j=1}^{n}\left(\widetilde{b}_{j}^{I}\right)_{(\alpha, \beta)} .
$$

The disparity (7) is considered as a necessary and sufficient condition for the existence of a feasible solution to PIF-MOFTP.

For a certain degree of $(\alpha, \beta)$-cut the PIF-MOFTP could be transformed into parametric $(\alpha, \beta)$-MOFTP as:

$$
\operatorname{Max} Z_{q}(x, \vartheta)=\frac{\sum_{i=1}^{m} \sum_{j=1}^{n}\left(c_{i j}+\vartheta \omega_{i j}\right)^{(q)} x_{i j}^{(q)}+\delta^{(q)}}{\sum_{i=1}^{m} \sum_{j=1}^{n} d_{i j}^{(q)} x_{i j}^{(q)}+\rho^{(q)}}, q=12, \ldots, Q,
$$

Subject to:

$$
\begin{gathered}
\sum_{j=1}^{n} x_{i j} \leq\left(a_{i}\right)_{(\alpha, \beta)} \quad i=1,2, \ldots, m, \\
\sum_{i=1}^{m} x_{i j} \geq\left(b_{j}\right)_{(\alpha, \beta)} \quad j=1,2, \ldots, n, \\
x_{i j} \geq 0, \quad i=1,2, \ldots, m, j=1,2, \ldots, n, \\
a_{i}^{L} \leq\left(a_{i}\right)_{(\alpha, \beta)} \leq a_{i}^{U}, \quad i=1,2, \ldots, m, \\
b_{j}^{L} \leq\left(b_{j}\right)_{(\alpha, \beta)} \leq b_{j}^{U}, \quad j=1,2, \ldots, n .
\end{gathered}
$$

Based on the concept of a convex linear combination method proposed in [40] parametric $(\alpha, \beta)$-MOFTP can be rewritten as:

$$
\operatorname{Max} Z_{q}(x, \vartheta)=\frac{\sum_{i=1}^{m} \sum_{j=1}^{n}\left(c_{i j}+\vartheta \omega_{i j}\right)^{(q)} x_{i j}^{(q)}+\delta^{(q)}}{\sum_{i=1}^{m} \sum_{j=1}^{n} d_{i j}^{(q)} x_{i j}^{(q)}+\rho^{(q)}}, q=12, \ldots, Q,
$$

\section{Subject to:}

$$
\begin{gathered}
\sum_{j=1}^{n} x_{i j} \leq \lambda a_{i}^{L}+(1-\lambda) a_{i}^{U} \quad i=1,2, \ldots, m, \\
\sum_{i=1}^{m} x_{i j} \geq \lambda b_{j}^{L}+(1-\lambda) b_{j}^{U} \quad j=1,2, \ldots, n, \\
x_{i j} \geq 0, \lambda \in[0,1], i=1,2, \ldots, m, j=1,2, \ldots, n,
\end{gathered}
$$

Let $M_{(\alpha, \beta)}$ denote the set of constraints in Equations (15)-(17), the parametric $(\alpha, \beta)$ MOFTP has an $(\alpha, \beta)$-Pareto optimal solution $x_{i j}^{*}$ at $\vartheta^{*}$. 
Definition 7. $(\alpha, \beta)$-Pareto optimal solution. $x_{i j}^{*} \in M_{(\alpha, \beta)}$ is said to be an $(\alpha, \beta)$-Pareto optimal solution to $(\alpha, \beta)$-MOFTP if and only if there does not exist another $x_{i j}^{\circ} \in M_{(\alpha, \beta)}$ $a_{i} \in\left(a_{i}\right)_{(\alpha, \beta)}, b_{j} \in\left(b_{j}\right)_{(\alpha, \beta)}$, such that $Z_{q}\left(x_{i j}^{\circ}, \vartheta^{*}\right) \geq Z_{q}\left(x_{i j}^{*}, \vartheta^{*}\right)$ with at least one strict inequality hold for $q(q=1,2, \ldots, Q)$.

\section{FGP Methodology for PIF-MOFTP}

In this section the FGP approach is applied to obtain the compromise solution of the parametric $(\alpha, \beta)$-MOFTP. The objective functions are modeled as fuzzy goals characterized by its' membership function $\mu_{\left(z_{q}\left(x, \theta^{*}\right)\right)}[36,44-46]$. The model formulation and solution process are carried out at $\vartheta=\vartheta^{*}$. The membership functions of the $q^{\text {th }}$ fuzzy goals $[36,44]$, is defined as:

$$
\mu_{\left(z_{q}\left(x, \vartheta^{*}\right)\right)}=\left\{\begin{array}{ll}
1, & \text { if } Z_{q}\left(x, \vartheta^{*}\right) \geq u_{q}{ }^{*}, \\
\frac{Z_{q}\left(x, \vartheta^{*}\right)-g_{q}{ }^{*}}{u_{q}{ }^{*}-g_{q}{ }^{*}}, & \text { if } g_{q}{ }^{*} \leq Z_{q}\left(x, \vartheta^{*}\right) \leq u_{q}{ }^{*}, \\
0, & \text { if } Z_{q}\left(x, \vartheta^{*}\right) \leq g_{q}{ }^{*},
\end{array} \quad q=1,2, \ldots, Q\right.
$$

where $u_{q}{ }^{*}=\max Z_{q}\left(\boldsymbol{x}, \vartheta^{*}\right), g_{q}{ }^{*}=\min Z_{q}\left(\boldsymbol{x}, \vartheta^{*}\right)$, and denotes the upper and lower tolerance limit for the membership function of $q^{\text {th }}$ objective, respectively. In the FGP approach, the most extensive level of membership is unity. So, the membership goals having the aspired level unity follows as [44]:

$$
\mu_{q}\left(Z_{q}\left(x, \vartheta^{*}\right)\right)+d_{q}^{-}-d_{q}^{+}=1, \quad q=1,2, \ldots, Q,
$$

where $d_{q}^{-}, d_{q}^{+} \geq 0$, with $d_{q}^{-} \times d_{q}^{+}=0$, denote the under- and over-deviations, respectively, from the aspired levels $[36,44]$. The final FGP model of the parametric $(\alpha, \beta)$-MOFTP can be obtained as:

$$
\operatorname{Min} A F=\sum_{q=1}^{Q} w_{q}^{-} d_{q}^{-},
$$

Subject to:

$$
\begin{gathered}
\frac{Z_{q}\left(x, \vartheta^{*}\right)-g_{q}{ }^{*}}{u_{q}{ }^{*}-g_{q}{ }^{*}}+d_{q}^{-}-d_{q}^{+}=1, \quad q=1,2, \ldots, Q, \\
\sum_{j=1}^{n} x_{i j} \leq \lambda a_{i}^{L}+(1-\lambda) a_{i}^{U} \quad i=1,2, \ldots, m, \\
\sum_{i=1}^{m} x_{i j} \geq \lambda b_{j}^{L}+(1-\lambda) b_{j}^{U} \quad j=1,2, \ldots, n, \\
x_{i j} \geq 0, \lambda \in[0,1], i=1,2, \ldots, m, j=1,2, \ldots, n, \\
d_{q}^{-} \times d_{q}^{+}=0, \text { and } d_{q}^{-}, d_{q}^{+} \geq 0, q=1,2, \ldots, Q,
\end{gathered}
$$

where $w_{q}^{-}$represents the relative importance of achieving the aspired levels of the respective fuzzy goals which given by [44,47]:

$$
w_{q}^{-}=\frac{1}{u_{q}{ }^{*}-g_{q}{ }^{*}}, \quad q=1,2, \ldots, Q
$$

Extension of Pal's Method to Linearize the Membership Goals

It can be easily realized that the parametric membership goals in Equation (19) are non-linear fractional in nature. To avoid such problem, Pal et al. [45] method is extended here to linearize the $q^{\text {th }}$ membership goals with single-scalar parameter $\vartheta=\vartheta^{*}$ as: 


$$
\begin{gathered}
\mu_{q}\left(Z_{q}\left(x, \vartheta^{*}\right)\right)+d_{q}^{-}-d_{q}^{+}=1, q=1,2, \ldots, Q, \\
L_{q}\left(Z_{q}\left(x, \vartheta^{*}\right)\right)-L_{q} g_{q}{ }^{*}+d_{q}^{-}-d_{q}^{+}=1 ; L_{q}=\frac{1}{u_{q}{ }^{*}-g_{i j}{ }^{*}}, \\
Z_{q}\left(x, \vartheta^{*}\right)=\frac{\sum_{i=1}^{m} \sum_{j=1}^{n}\left(c_{i j}+\vartheta^{*} \omega_{i j}\right)^{(q)} x_{i j}^{(q)}+\delta^{(q)}}{\sum_{i=1}^{m} \sum_{j=1}^{n} d_{i j}^{(q)} x_{i j}^{(q)}+\rho^{(q)}}, q=1,2, \ldots, Q,
\end{gathered}
$$

Substituting from Equation (29) in Equation (28), we obtain:

$$
\begin{aligned}
& L_{q} \frac{\sum_{i=1}^{m} \sum_{j=1}^{n}\left(c_{i j}+\vartheta^{*} \omega_{i j}\right)^{(q)} x_{i j}^{(q)}+\delta^{(q)}}{\sum_{i=1}^{m} \sum_{j=1}^{n} d_{i j}^{(q)} x_{i j}^{(q)}+\rho^{(q)}}-L_{q} g_{q}{ }^{*}+d_{q}^{-}-d_{q}^{+}=1, \\
& L_{q}\left[\sum_{i=1}^{m} \sum_{j=1}^{n}\left(c_{i j}+\vartheta^{*} \omega_{i j}\right)^{(q)} x_{i j}^{(q)}+\delta^{(q)}\right]-L_{q} g_{q}{ }^{*}\left[\sum_{i=1}^{m} \sum_{j=1}^{n} d_{i j}^{(q)} x_{i j}^{(q)}+\rho^{(q)}\right]+d_{q}^{-}\left[\sum_{i=1}^{m} \sum_{j=1}^{n} d_{i j}^{(q)} x_{i j}^{(q)}+\rho^{(q)}\right] \\
& -d_{q}^{+}\left[\sum_{i=1}^{m} \sum_{j=1}^{n} d_{i j}^{(q)} x_{i j}^{(q)}+\rho^{(q)}\right]=\left[\sum_{i=1}^{m} \sum_{j=1}^{n} d_{i j}^{(q)} x_{i j}^{(q)}+\rho^{(q)}\right] \text {, } \\
& \left(\begin{array}{l}
L_{q}\left[\sum_{i=1}^{m} \sum_{j=1}^{n}\left(c_{i j}+\vartheta^{*} \omega_{i j}\right)^{(q)} x_{i j}^{(q)}+\delta^{(q)}\right] \\
+d_{q}^{-}\left[\sum_{i=1}^{m} \sum_{j=1}^{n} d_{i j}^{(q)} x_{i j}^{(q)}+\rho^{(q)}\right] \\
-d_{q}^{+}\left[\sum_{i=1}^{m} \sum_{j=1}^{n} d_{i j}^{(q)} x_{i j}^{(q)}+\rho^{(q)}\right]
\end{array}\right)=\left(1+L_{q} g_{q^{*}}{ }^{(q)}\left[\sum_{i=1}^{m} \sum_{j=1}^{n} d_{i j}^{(q)} x_{i j}^{(q)}+\rho^{(q)}\right],\right. \\
& \left(\begin{array}{l}
L_{q}\left[\sum_{i=1}^{m} \sum_{j=1}^{n}\left(c_{i j}+\vartheta^{*} \omega_{i j}\right)^{(q)} x_{i j}^{(q)}+\delta^{(q)}\right] \\
+d_{q}^{-}\left[\sum_{i=1}^{m} \sum_{j=1}^{n} d_{i j}^{(q)} x_{i j}^{(q)}+\rho^{(q)}\right] \\
-d_{q}^{+}\left[\sum_{i=1}^{m} \sum_{j=1}^{n} d_{i j}^{(q)} x_{i j}^{(q)}+\rho^{(q)}\right]
\end{array}\right)=L_{q}^{\circ}\left[\sum_{i=1}^{m} \sum_{j=1}^{n} d_{i j}^{(q)} x_{i j}^{(q)}+\rho^{(q)}\right] ; \quad L_{q}^{0}=\left(1+L_{q} g_{q}{ }^{*}\right) \\
& {\left[L_{q} \sum_{i=1}^{m} \sum_{j=1}^{n}\left(c_{i j}+\vartheta^{*} \omega_{i j}\right)^{(q)}-L_{q}^{0} \sum_{i=1}^{m} \sum_{j=1}^{n} d_{i j}^{(q)}\right] x_{i j}^{(q)}+d_{q}^{-}\left[\sum_{i=1}^{m} \sum_{j=1}^{n} d_{i j}^{(q)} x_{i j}^{(q)}+\rho^{(q)}\right]-d_{q}^{+}\left[\sum_{i=1}^{m} \sum_{j=1}^{n} d_{i j}^{(q)} x_{i j}^{(q)}+\rho^{(q)}\right]} \\
& =\left[L_{q}^{0} \rho^{(q)}-L_{q} \delta^{(q)}\right] \text {, } \\
& C_{i j}^{(q)} x_{i j}^{(q)}+d_{q}^{-}\left[\sum_{i=1}^{m} \sum_{j=1}^{n} d_{i j}^{(q)} x_{i j}^{(q)}+\rho^{(q)}\right]-d_{q}^{+}\left[\sum_{i=1}^{m} \sum_{j=1}^{n} d_{i j}^{(q)} x_{i j}^{(q)}+\rho^{(q)}\right]=G_{q} ;
\end{aligned}
$$

where

$$
\begin{gathered}
C_{i j}^{(q)}=\left[L_{q} \sum_{i=1}^{m} \sum_{j=1}^{n}\left(c_{i j}+\vartheta^{*} \omega_{i j}\right)^{(q)}-L_{q}^{0} \sum_{i=1}^{m} \sum_{j=1}^{n} d_{i j}^{(q)}\right], \\
G_{q}=\left[L_{q}^{0} \rho^{(q)}-L_{q} \delta^{(q)}\right],
\end{gathered}
$$


Considering Pal et al. [45], the goal expression in Equation (35) can be linearized as follows. Letting $D_{q}^{-}=d_{q}^{-}\left[\sum_{i=1}^{m} \sum_{j=1}^{n} d_{i j}^{(q)} x_{i j}^{(q)}+\rho^{(q)}\right]$ and $D_{q}^{+}=d_{q}^{+}\left[\sum_{i=1}^{m} \sum_{j=1}^{n} d_{i j}^{(q)} x_{i j}^{(q)}+\rho^{(q)}\right]$, then the linear form of expression in Equation (32) is obtained as:

$$
C_{i j}^{(q)} x_{i j}^{(q)}+D_{q}^{-}-D_{q}^{+}=G_{q},
$$

with $D_{q}^{-}, D_{q}^{+} \geq 0$; and $D_{q}^{-} \times D_{q}^{+}=0$, since $d_{q}^{-}, d_{q}^{+} \geq 0$, and $\sum_{i=1}^{m} \sum_{j=1}^{n} d_{i j}^{(q)} x_{i j}^{(q)}+\rho^{(q)}>0$. So, minimization of $d_{q}^{-}$means minimization of $D_{q}^{-}=d_{q}^{-}\left[\sum_{i=1}^{m} \sum_{j=1}^{n} d_{i j}^{(q)} x_{i j}^{(q)}+\rho^{(q)}\right]$ which is also non-linear. So, involvement of $d_{q}^{-} \leq 1$, in the solution leads to impose the following constraint in the model:

$$
\frac{D_{q}^{-}}{\left[\sum_{i=1}^{m} \sum_{j=1}^{n} d_{i j}^{(q)} x_{i j}^{(q)}+\rho^{(q)}\right]} \leq 1
$$

Now, the final FGP model of the parametric $(\alpha, \beta)$-MOFTP in model (20)-(25) becomes:

$$
\operatorname{Min} A F=\sum_{q=1}^{Q} w_{q}^{-} d_{q}^{-}
$$

Subject to:

$$
\begin{gathered}
{\left[L_{q} \sum_{i=1}^{m} \sum_{j=1}^{n}\left(c_{i j}+\vartheta^{*} \omega_{i j}\right)^{(q)}-L_{q}^{0} \sum_{i=1}^{m} \sum_{j=1}^{n} d_{i j}^{(q)}\right] x_{i j}^{(q)}+D_{q}^{-}-D_{q}^{+}=\left[L_{q}^{0} \rho^{(q)}-L_{q} \delta^{(q)}\right],} \\
\sum_{i=1}^{m} \sum_{j=1}^{n}-d_{i j}^{(q)} x_{i j}^{(q)}+D_{q}^{-} \leq \rho^{(q)}, q=1,2, \ldots, Q, \forall i, j, \\
\sum_{j=1}^{n} x_{i j} \leq \lambda a_{i}^{L}+(1-\lambda) a_{i}^{U}, i=1,2, \ldots, m, \\
\sum_{i=1}^{m} x_{i j} \geq \lambda b_{j}^{L}+(1-\lambda) b_{j}^{U} j=1,2, \ldots, n, \\
x_{i j} \geq 0, \lambda \in[0,1], i=1,2, \ldots, m, j=1,2, \ldots, n, \\
D_{q}^{-} \times D_{q}^{+}=0, \text { and } D_{q}^{-}, D_{q}^{+} \geq 0, q=1,2, \ldots, Q .
\end{gathered}
$$

Thus, the above FGP model provides the satisfactory solution $x_{i j}^{*}$ for the parametric $(\alpha, \beta)$-MOFTP.

\section{The SSFK for Parametric $(\alpha, \beta)$-MOFTP}

The main area of inquiry is as follows: having solved the parametric $(\alpha, \beta)$-MOFTP, to what extent can its data with respect to $\alpha, \beta$ and $\vartheta$ be changed without invalidating the efficiency of its $(\alpha, \beta)$-Pareto optimal solution? The set of feasible parameters, the solvability set, and the SSFK for parametric $(\alpha, \beta)$-MOFTP are defined as:

Definition 8. The set of feasible parameters for the parametric $(\alpha, \beta)$-MOFTP is defined by:

$$
\mathcal{F}=\left\{\begin{array}{c|c}
a \in R^{m} & a_{i} \in L_{\alpha, \beta}\left(\widetilde{a}_{i}^{I}\right), i=1,2, \ldots m ; b_{j} \in L_{\alpha, \beta}\left(\widetilde{b}_{j}^{I}\right), j=1,2, \ldots, n ; \\
b \in R^{n} & \alpha, \beta \in[0,1] ; \text { and } M_{(\alpha, \beta)}\left(x_{i j}, a, b\right) \neq \varnothing
\end{array}\right\} .
$$

Definition 9. The solvability set $\mathcal{M}$ of the parametric $(\alpha, \beta)$-MOFTP is defined by: 


$$
\mathcal{M}=\left\{\begin{array}{ll}
(\vartheta, a, b) \in R \times R^{m} \times R^{n} \mid \begin{array}{c}
\text { parametric }(\alpha, \beta)-\text { MOFTP has } \\
\text { an }(\alpha, \beta)-\text { Pareto optimal solution. }
\end{array}
\end{array}\right\} .
$$

Definition 10. Suppose that $x_{i j}^{*}$ be an $(\alpha, \beta)$-Pareto optimal solution of the parametric $(\alpha, \beta)$ MOFTP, then the SSFK $S_{1}\left(x_{i j}^{*}, \alpha, \beta\right)$ corresponding to $x_{i j}^{*}$ is defined by:

$S_{1}\left(x_{i j}^{*}, \alpha, \beta\right)=\left\{(\vartheta, a, b) \in R \times R^{m} \times R^{n} \mid \begin{array}{c}x_{i j}^{*} \text { is an }(\alpha, \beta)-\text { Pareto optimal solution of } \\ \text { parametric }(\alpha, \beta)-\text { MOFTP }\end{array}\right\}$.

The SSFK of the parametric $(\alpha, \beta)$-MOFTP is the set of all parameters corresponding to one $(\alpha, \beta)$-Pareto optimal solution $[35,36]$. It is easy to see that the stability of the parametric $(\alpha, \beta)$-MOFTP model (14)-(17) implies the stability of the parametric FGP model which is defined as follows:

$$
\operatorname{Min} A F=\sum_{q=1}^{Q} w_{q}^{-} d_{q}^{-}, q
$$

\section{Subject to:}

$$
\begin{gathered}
{\left[L_{q} \sum_{i=1}^{m} \sum_{j=1}^{n}\left(c_{i j}+\vartheta \omega_{i j}\right)^{(q)}-L_{q}^{0} \sum_{i=1}^{m} \sum_{j=1}^{n} d_{i j}^{(q)}\right] x_{i j}^{(q)}+D_{q}^{-}-D_{q}^{+}=\left[L_{q}^{0} \rho^{(q)}-L_{q} \delta^{(q)}\right],} \\
\sum_{i=1}^{m} \sum_{j=1}^{n}-d_{i j}^{(q)} x_{i j}^{(q)}+D_{q}^{-} \leq \rho^{(q)}, q=1,2, \ldots, Q, \forall i, j \\
\sum_{j=1}^{n} x_{i j} \leq \lambda a_{i}^{L}+(1-\lambda) a_{i}^{U}, \quad i=1,2, \ldots, m, \\
\sum_{i=1}^{m} x_{i j} \geq \lambda b_{j}^{L}+(1-\lambda) b_{j}^{U}, \quad j=1,2, \ldots, n, \\
x_{i j} \geq 0, \lambda \in[0,1], \vartheta \in R, i=1,2, \ldots, m, j=1,2, \ldots, n, \\
D_{q}^{-} \times D_{q}^{+}=0, \text { and } D_{q}^{-}, D_{q}^{+} \geq 0, q=1,2, \ldots, Q .
\end{gathered}
$$

\subsection{KKT Optimality Conditions for Parametric FGP Model}

The Lagrangian function of parametric FGP model (47)-(53) follows as [36,37]:

$$
\begin{aligned}
L=\left[\sum_{q=1}^{Q} w_{q}^{-} D_{q}^{-}\right] & +\xi_{q}\left[\left[L_{q} \sum_{i=1}^{m} \sum_{j=1}^{n}\left(c_{i j}+\vartheta \omega_{i j}\right)^{(q)}-L_{q}^{0} \sum_{i=1}^{m} \sum_{j=1}^{n} d_{i j}^{(q)}\right] x_{i j}^{(q)}+D_{q}^{-}-D_{q}^{+}-\left[L_{q}^{0} \rho^{(q)}-L_{q} \delta^{(q)}\right]\right] \\
& +v_{q}\left[\sum_{i=1}^{m} \sum_{j=1}^{n}-d_{i j}^{(q)} x_{i j}^{(q)}+D_{q}^{-}-\rho^{(q)}\right]+\tau_{i}\left[\sum_{j=1}^{n} x_{i j}-\left(\lambda a_{i}^{L}+(1-\lambda) a_{i}^{U}\right)\right] \\
& +\eta_{j}\left[-\sum_{i=1}^{m} x_{i j}+\left(\lambda b_{j}^{L}+(1-\lambda) b_{j}^{U}\right)\right]+\varphi_{i j}\left[-x_{i j}\right]+\psi_{i}\left[-a_{i}^{L}\right]+\phi_{j}\left[-b_{j}^{L}\right]+\omega_{i}\left[-a_{i}^{U}\right]+\epsilon_{j}\left[-b_{j}^{U}\right] \\
& +\zeta_{q}\left[-D_{q}^{-}\right]+\pi_{q}\left[-D_{q}^{+}\right],
\end{aligned}
$$

where $\xi, v, \tau, \eta, \varphi, \psi, \phi, \omega, \epsilon, \zeta$ and $\pi$ are the Lagrange multipliers. Thus, KKT optimality conditions $[28,36,37,39]$ have the following form:

$$
\begin{gathered}
\frac{\partial L}{\partial x_{i j}}=\xi_{q}\left[L_{q} \sum_{i=1}^{m} \sum_{j=1}^{n}\left(c_{i j}+\vartheta \omega_{i j}\right)^{(q)}-L_{q}^{0} \sum_{i=1}^{m} \sum_{j=1}^{n} d_{i j}^{(q)}\right]+v_{q}\left[\sum_{i=1}^{m} \sum_{j=1}^{n}-d_{i j}^{(q)}\right]+\tau_{i}-\eta_{j}-\varphi_{i j}=0, i=1,2, \ldots, m, j=1,2, \ldots, n, \\
\frac{\partial L}{\partial a_{i}^{L}}=-\lambda \tau_{i}-\psi_{i}=0, i=1,2, \ldots m,
\end{gathered}
$$




$$
\begin{aligned}
& \frac{\partial L}{\partial a_{i}^{U}}=-(1-\lambda) \tau_{i}-\omega_{i}=0, i=1,2, \ldots m, \\
& \frac{\partial L}{\partial b_{j}^{L}}=\lambda \eta_{j}-\phi_{j}=0, \quad i=1,2, \ldots m, \\
& \frac{\partial L}{\partial b_{j}^{U}}=(1-\lambda) \eta_{j}-\epsilon_{j}=0, i=1,2, \ldots m, \\
& \frac{\partial L}{\partial D_{q}^{-}}=\sum_{q=1}^{Q} w_{q}^{-}+\xi_{q}+v_{q}-\zeta_{q}=0, q=1,2, \ldots, Q \text {, } \\
& \frac{\partial L}{\partial D_{q}^{+}}=-\xi_{q}-\pi_{q}=0, \quad q=1,2, \ldots, Q, \\
& {\left[L_{q} \sum_{i=1}^{m} \sum_{j=1}^{n}\left(c_{i j}+\vartheta \omega_{i j}\right)^{(q)}-L_{q}^{0} \sum_{i=1}^{m} \sum_{j=1}^{n} d_{i j}^{(q)}\right] x_{i j}^{(q)}+D_{q}^{-}-D_{q}^{+}-\left[L_{q}^{0} \rho^{(q)}-L_{q} \delta^{(q)}\right]=0,} \\
& \sum_{i=1}^{m} \sum_{j=1}^{n}-d_{i j}^{(q)} x_{i j}^{(q)}+D_{q}^{-}-\rho^{(q)} \leq 0, q=1,2, \ldots, Q, \forall i, j \\
& \sum_{j=1}^{n} x_{i j}-\left[\lambda a_{i}^{L}+(1-\lambda) a_{i}^{U}\right] \leq 0, \quad i=1,2, \ldots, m, \\
& {\left[\lambda b_{j}^{L}+(1-\lambda) b_{j}^{U}\right]-\sum_{i=1}^{m} x_{i j} \leq 0, j=1,2, \ldots, n,} \\
& x_{i j} \geq 0, i=1,2, \ldots, m, j=1,2, \ldots, n, \\
& D_{i j}^{-}, D_{i j}^{+} \geq 0, q=1,2, \ldots, Q, \\
& v_{q}\left[\sum_{i=1}^{m} \sum_{j=1}^{n}-d_{i j}^{(q)} x_{i j}^{(q)}+D_{q}^{-}-\rho^{(q)}\right]=0, q=1,2, \ldots, Q, \forall i, j \\
& \tau_{i}\left[\sum_{j=1}^{n} x_{i j}-\left(\lambda a_{i}^{L}+(1-\lambda) a_{i}^{U}\right)\right]=0, \quad i=1,2, \ldots, m, \\
& \eta_{j}\left[-\sum_{i=1}^{m} x_{i j}+\left(\lambda b_{j}^{L}+(1-\lambda) b_{j}^{U}\right)\right]=0, j=1,2, \ldots, n, \\
& \varphi_{i j}\left[x_{i j}\right]=0 \text {, } \\
& \psi_{i}\left[a_{i}^{L}\right]=0 \text {, } \\
& \phi_{j}\left[b_{j}^{L}\right]=0, \\
& \omega_{i}\left[a_{i}^{U}\right]=0 \text {, } \\
& \epsilon_{j}\left[b_{j}^{U}\right]=0 \text {, } \\
& \zeta_{q}\left[D_{q}^{-}\right]=0 \text {, } \\
& \pi_{q}\left[D_{q}^{+}\right]=0,
\end{aligned}
$$

$v, \tau, \eta, \varphi, \psi, \phi, \omega, \epsilon, \zeta, \pi \geq 0$, and $\vartheta, \xi \in R$; 
where the KKT conditions (55)-(78) are evaluated at $x_{i j}^{*}$. Solving the system of Equations (55)-(78), the SSFK $S_{1}\left(x_{i j}^{*}, \alpha, \beta\right)$ for parametric IF-MOFTP is obtained.

\subsection{Algorithm for Determination of the SSFK $S_{1}\left(x_{i j}^{*}, a, b\right)$}

Following the above discussion, the algorithm for obtaining the $\operatorname{SSFK} S_{1}\left(x_{i j^{\prime}}^{*} \alpha, \beta\right)$ for parametric $(\alpha, \beta)$-MOFTP van be described as follows (Algorithms 1 and 2):
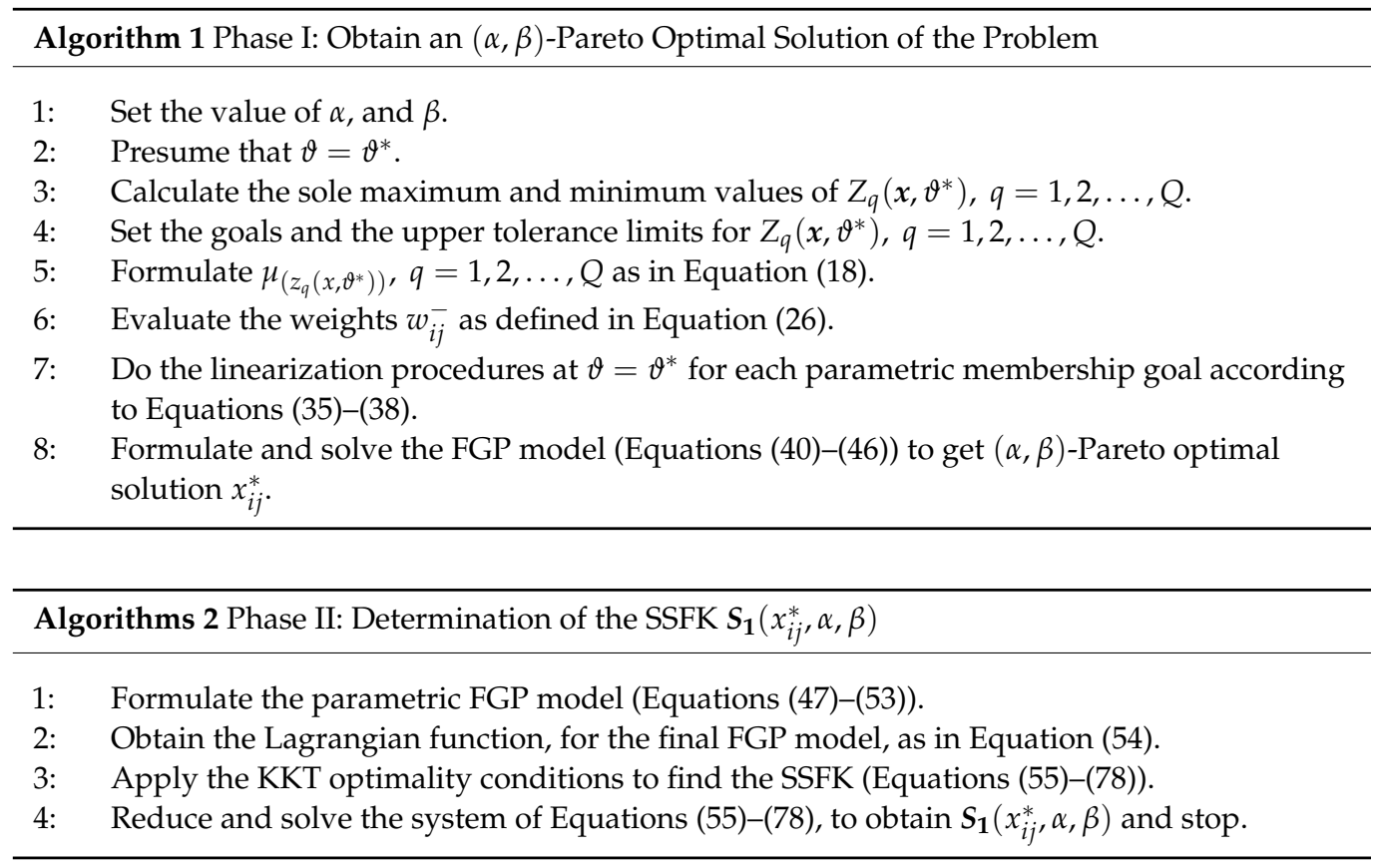

\section{Numerical Example}

To demonstrate the proposed algorithm for finding the SSFK, consider the following parametric IF-MOFTP:

$$
\operatorname{Max}\left(\begin{array}{c}
Z_{1}(x, \vartheta)=\frac{\vartheta x_{11}+(2+\vartheta) x_{12}+(3+2 \vartheta) x_{21}+6 x_{22}+4}{x_{11}+3 x_{12}+x_{21}+2 x_{22}+2} \\
Z_{2}(x, \vartheta)=\frac{2 x_{11}+(3+\vartheta) x_{12}+(4+2 \vartheta) x_{21}+(5+\vartheta) x_{22}+6}{x_{11}+2 x_{12}+3 x_{21}+x_{22}+4}
\end{array}\right)
$$

\section{Subject to:}

Supply constraints:

$$
x_{11}+x_{12} \leq \widetilde{a}_{1}^{I}, x_{21}+x_{22} \leq \widetilde{a}_{2}^{I}
$$

Demand constraints:

$$
x_{11}+x_{21} \geq \widetilde{b}_{1}^{I}, x_{12}+x_{22} \geq \widetilde{b}_{2}^{I},
$$

where the membership functions $\mu_{\widetilde{a}_{1}^{I}}(x), \mu_{\widetilde{a}_{2}^{I}}(x), \mu_{\widetilde{b}_{1}^{I}}(x), \mu_{\widetilde{b}_{2}^{I}}(x)$ and the non-membership functions $\gamma_{\widetilde{a}_{1}^{I}}(x), \gamma_{\widetilde{a}_{2}^{I}}(x), \gamma_{\widetilde{b}_{2}^{I}}(x), \gamma_{\widetilde{b}_{2}^{I}}(x)$ of the supplies and demands are described as follows:

$$
\mu_{\widetilde{a}_{1}^{I}}(x)= \begin{cases}\frac{x-140}{20} & \text { if } 140 \leq x \leq 160, \\
\frac{180-x}{20} & \text { if } 160 \leq x \leq 180, \gamma_{\widetilde{a}_{1}^{I}}(x)=\left\{\begin{array}{ll}
\frac{160-x}{30} & \text { if } 130 \leq x \leq 160, \\
0 & \text { otherwise, }
\end{array} \text { if } 160 \leq x \leq 200,\right. \\
1 \quad & \text { otherwise, }\end{cases}
$$




$$
\begin{aligned}
& \mu_{\widetilde{a}_{2}^{I}}(x)= \begin{cases}\frac{x-220}{20} & \text { if } 220 \leq x \leq 240, \\
\frac{250-x}{10} & \text { if } 240 \leq x \leq 250, \gamma_{\widetilde{a}_{2}^{I}}(x)=\left\{\begin{array}{ll}
\frac{240-x}{20} & \text { if } 210 \leq x \leq 240, \\
\frac{x-240}{30} & \text { if } 240 \leq x \leq 270, \\
1 & \text { otherwise, }
\end{array}\right. \text { otherwise, }\end{cases}
\end{aligned}
$$

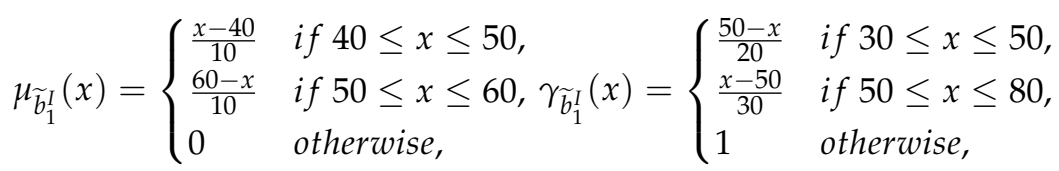

$$
\begin{aligned}
& \mu_{\widetilde{b}_{2}^{I}}(x)= \begin{cases}\frac{x-310}{10} & \text { if } 310 \leq x \leq 320, \\
\frac{350-x}{30} & \text { if } 320 \leq x \leq 350, \gamma_{\widetilde{b}_{2}^{I}}(x)=\left\{\begin{array}{ll}
\frac{320-x}{20} & \text { if } 300 \leq x \leq 320, \\
\frac{x-320}{60} & \text { if } 320 \leq x \leq 380, \\
1 & \text { otherwise, }
\end{array}\right. \text { otherwise, }\end{cases}
\end{aligned}
$$

Phase I: Finding an $(\alpha, \beta)$-Pareto optimal solution of the parametric IF-MOFTP.

For a desired values of $\alpha=0.6$, and $\beta=0.2$, then applying the concept of $(\alpha, \beta)$-cut of the IFN we formulate the $(\alpha, \beta)$-MOFTP at $\vartheta=\vartheta^{*}=3$.

$$
\operatorname{Max}\left(\begin{array}{c}
Z_{1}(x)=\frac{3 x_{11}+5 x_{12}+9 x_{21}+6 x_{22}+8}{x_{11}+3 x_{12}+x_{21}+2 x_{22}+2}, \\
Z_{2}(x)=\frac{2 x_{11}+6 x_{12}+10 x_{21}+8 x_{22}+6}{x_{11}+2 x_{12}+3 x_{21}+x_{22}+4}
\end{array}\right),
$$

\section{Subject to:}

Supply constraints:

$$
x_{11}+x_{12} \leq[154,168], x_{21}+x_{22} \leq[234,244] .
$$

Demand constraints:

$$
x_{11}+x_{21} \geq[46,54], x_{12}+x_{22} \geq[316,332] .
$$

Based on the concept of convex linear combination on the constraints, then we obtain the MOFTP:

$$
\operatorname{Max}\left(\begin{array}{c}
Z_{1}(x)=\frac{3 x_{11}+5 x_{12}+9 x_{21}+6 x_{22}+8}{x_{11}+3 x_{12}+x_{21}+2 x_{22}+2} \\
Z_{2}(x)=\frac{2 x_{11}+6 x_{12}+10 x_{21}+8 x_{22}+6}{x_{11}+2 x_{12}+3 x_{21}+x_{22}+4}
\end{array}\right),
$$

\section{Subject to:}

$$
x_{11}+x_{12} \leq 165.2, x_{21}+x_{22} \leq 240, x_{11}+x_{21} \geq 51.6, x_{12}+x_{22} \geq 328.8 .
$$

A FGP approach is utilized to solve the MOFTP according to the model of Equations (40)-(46). Firstly, the coefficients of the linearized membership goals are obtained in Table 1.

Table 1. The coefficient of the linearized membership goals $\left(c^{i j}\right)^{T}$ and $G_{i j}$.

\begin{tabular}{ccc}
\hline & $Z_{\mathbf{1}}(\boldsymbol{x})$ & $\boldsymbol{Z}_{\mathbf{2}}(\boldsymbol{x})$ \\
\hline$\left(c_{i j}^{q}\right)^{T}$ & $\left(\begin{array}{c}0.682 \\
-10.22 \\
19.081 \\
1.364\end{array}\right)^{T}$ & $\left(\begin{array}{c}-2.8628 \\
-4.048 \\
-5.234 \\
G_{i j}\end{array}\right.$ \\
-7.497 & 2.1688 \\
$G_{i j}$ & 13.128 \\
\hline
\end{tabular}

$$
\operatorname{Min} A F=3.0665 D_{1}^{-}+0.8386 D_{2}^{-} \text {, }
$$

\section{Subject to:}

$$
\begin{aligned}
& 0.682 x_{11}-10.22 x_{12}+19.081 x_{21}+1.364 x_{22}+D_{1}^{-}-D_{1}^{+}=-7.497 \\
& -2.8628 x_{11}-4.048 x_{12}-5.234 x_{21}+2.169 x_{22}+D_{2}^{-}-D_{2}^{+}=13.128
\end{aligned}
$$




$$
\begin{gathered}
-x_{11}-3 x_{12}-x_{21}-2 x_{22}+D_{1}^{-} \leq 2, \\
-x_{11}-2 x_{12}-3 x_{21}-x_{22}+D_{2}^{-} \leq 4, \\
x_{11}+x_{12} \leq 165.2, \\
x_{21}+x_{22} \leq 240, \\
x_{11}+x_{21} \geq 51.6, \\
x_{12}+x_{22} \geq 328.8, \\
x_{11}, x_{12}, x_{21}, x_{22}, D_{1}^{-}, D_{1}^{+}, D_{2}^{-}, D_{2}^{+} \geq 0 .
\end{gathered}
$$

Using Lingo programming, the $(\alpha, \beta)$-Pareto optimal solution of the parametric IF-

\begin{tabular}{|c|c|c|}
\hline & $Z_{1}(x, \vartheta)$ & $Z_{2}(x, \vartheta)$ \\
\hline $\begin{array}{c}{\left[\left(c_{i j}+\vartheta \omega_{i j}\right)^{(q)}\right]^{T}} \\
G_{i j}\end{array}$ & $\left.\begin{array}{c}-8.518+3.067 \vartheta \\
-19.42+3.067 \vartheta \\
0.682+6.133 \vartheta \\
1.364 \\
-7.497\end{array}\right)^{T}$ & $\begin{array}{c}-2.863 \\
-6.564+0.839 \vartheta \\
-10.266+1.677 \vartheta \\
-0.347+0.839 \vartheta \\
13.128\end{array}$ \\
\hline
\end{tabular}
MOFTP is obtained at $\left(x_{11}^{*}, x_{12}^{*}, x_{21}^{*}, x_{22}^{*}, D_{1}^{-}, D_{1}^{+}, D_{2}^{-}, D_{2}^{+}\right)=(0,165.88,76.39,163.61,0$, $0,726.78,0)$.

Phase II: determination of the SSFK $S_{1}\left(x^{*}, \alpha, \beta\right)$.

To determine the SSFK $S_{1}\left(x^{*}, a, b\right)$ of the parametric IF-MOFTP, the coefficients of the linearized membership goals in the parametric form are recalculated in Table 2.

Table 2. The coefficients of the linearized membership goals $\left[\left(c_{i j}+\vartheta \omega_{i j}\right)^{(q)}\right]^{T}$ and $G_{i j}$.

Therefore, the stability of parametric IF-MOFTP implies the stability of the parametric FGP model which is defined as:

$$
\operatorname{Min} A F=3.067 D_{1}^{-}+0.839 D_{2}^{-},
$$

\section{Subject to:}

$$
\begin{gathered}
(-8.518+3.067 \vartheta) x_{11}+(-19.42+3.067 \vartheta) x_{12}+(0.682+6.133 \vartheta) x_{21}+1.364 x_{22} \\
+D_{1}^{-}-D_{1}^{+}=-7.497, \\
-2.8628 x_{11}+(-6.564+0.839 \vartheta) x_{12}+(-10.266+1.677 \vartheta) x_{21} \\
+(-0.347+0.839 \vartheta) x_{22}+D_{2}^{-}-D_{2}^{+}=13.128, \\
-x_{11}-3 x_{12}-x_{21}-2 x_{22}+D_{1}^{-} \leq 2, \\
-x_{11}-2 x_{12}-3 x_{21}-x_{22}+D_{2}^{-} \leq 4, \\
x_{11}+x_{12} \leq 0.2 a_{1}^{L}+0.8 a_{1}^{U}, \\
x_{21}+x_{22} \leq 0.4 a_{2}^{L}+0.6 a_{2}^{U}, \\
x_{11}+x_{21} \geq 0.3 b_{1}^{L}+0.7 b_{1}^{U}, \\
x_{12}+x_{22} \geq 0.2 b_{2}^{L}+0.8 b_{2}^{U}, \\
x_{11}, x_{12}, x_{21}, x_{22}, a_{1}^{L}, a_{1}^{U}, a_{2}^{L}, a_{2}^{U}, b_{1}^{L}, b_{1}^{U}, b_{2}^{L}, b_{2}^{U} \geq 0, \\
D_{1}^{-}, D_{1}^{+}, D_{2}^{-}, D_{2}^{+} \geq 0 ; \vartheta \in R
\end{gathered}
$$

The Lagrangean function of the above parametric FGP model follows as: 


$$
\begin{aligned}
& L \\
& =3.067 D_{1}^{-}+0.839 D_{2}^{-}+\xi_{1}\left[\begin{array}{c}
(-8.518+3.067 \vartheta) x_{11}+(-19.42+3.067 \vartheta) x_{12} \\
+(0.682+6.133 \vartheta) x_{21}+1.364 x_{22}+D_{1}^{-}-D_{1}^{+}+7.497
\end{array}\right] \\
& +\xi_{2}\left[\begin{array}{c}
-2.8628 x_{11}+(-6.564+0.839 \vartheta) x_{12}+(-10.266+1.677 \vartheta) x_{21} \\
+(-0.347+0.839 \vartheta) x_{22}+D_{2}^{-}-D_{2}^{+}-13.128
\end{array}\right] \\
& +\vartheta_{1}\left[-x_{11}-3 x_{12}-x_{21}-2 x_{22}+D_{1}^{-}-2\right]+\vartheta_{2}\left[-x_{11}-2 x_{12}-3 x_{21}-x_{22}+D_{2}^{-}-4\right] \\
& +\tau_{1}\left[x_{11}+x_{12}-0.2 a_{1}^{L}-0.8 a_{1}^{U}\right]+\tau_{2}\left[x_{21}+x_{22}-0.4 a_{2}^{L}-0.6 a_{2}^{U}\right] \\
& +\eta_{1}\left[-x_{11}-x_{21}+0.3 b_{1}^{L}+0.7 b_{1}^{U}\right]+\eta_{2}\left[-x_{12}-x_{22}+0.2 b_{2}^{L}+0.8 b_{2}^{U}\right]+\varphi_{1}\left[-x_{11}\right] \\
& +\varphi_{2}\left[-x_{12}\right]+\varphi_{3}\left[-x_{21}\right]+\varphi_{4}\left[-x_{22}\right]+\psi_{1}\left[-a_{1}^{L}\right]+\psi_{2}\left[-a_{2}^{L}\right]+\phi_{1}\left[-b_{1}^{L}\right]+\phi_{2}\left[-b_{2}^{L}\right] \\
& +\omega_{1}\left[-a_{1}^{U}\right]+\omega_{2}\left[-b_{2}^{U}\right]+\epsilon_{1}\left[-b_{1}^{U}\right]+\epsilon_{2}\left[-b_{2}^{U}\right]+\zeta_{1}\left[-D_{1}^{-}\right]+\zeta_{2}\left[-D_{2}^{-}\right]+\pi_{1}\left[-D_{1}^{+}\right] \\
& +\pi_{2}\left[-D_{2}^{+}\right]
\end{aligned}
$$

where $\vartheta, \xi_{1}, \xi_{2} \in R$, and $v_{1}, v_{2}, \tau_{1}, \tau_{2}, \eta_{1}, \eta_{2}, \varphi_{1}, \varphi_{2}, \varphi_{3}, \varphi_{4}, \psi_{1}, \psi_{2}, \phi_{1}, \phi_{2}, \omega_{1}, \omega_{2}, \epsilon_{1}, \epsilon_{2} \geq 0$, and $\zeta_{1}, \zeta_{2}, \pi_{1}, \pi_{2} \geq 0$, are the Lagrange multipliers. Therefore, KKT optimality conditions follows as:

$$
\begin{gathered}
\frac{\partial L}{\partial x_{11}}=(-8.518+3.067 \vartheta) \xi_{1}-2.863 \xi_{2}-v_{1}-v_{2}+\tau_{1}-\eta_{1}-\varphi_{1}=0 \\
\frac{\partial L}{\partial x_{12}}=(-19.42+3.067 \vartheta) \xi_{1}+(-6.564+0.839 \vartheta) \xi_{2}-3 v_{1}-2 v_{2}+\tau_{1}-\eta_{2}-\varphi_{2}=0 \\
\frac{\partial L}{\partial x_{21}}=(0.682+6.133 \vartheta) \xi_{1}+(-10.266+1.677 \vartheta) \xi_{2}-v_{1}-3 v_{2}+\tau_{2}-\eta_{1}-\varphi_{3}=0 \\
\frac{\partial L}{\partial x_{22}}=1.364 \xi_{1}+(-0.347+0.839 \vartheta) \xi_{2}-2 v_{1}-v_{2}+\tau_{2}-\eta_{2}-\varphi_{4}=0 \\
\frac{\partial L}{\partial a_{1}^{L}}=-0.2 \tau_{1}-\psi_{1}=0 \\
\frac{\partial L}{\partial a_{1}^{U}}=-0.8 \tau_{1}-\omega_{1}=0 \\
\frac{\partial L}{\partial a_{2}^{L}}=-0.4 \tau_{2}-\psi_{2}=0 \\
\frac{\partial L}{\partial a_{2}^{U}}=-0.6 \tau_{2}-\omega_{2}=0 \\
\frac{\partial L}{\partial b_{1}^{L}}=0.3 \eta_{1}-\phi_{1}=0 \\
\frac{\partial L}{\partial b_{1}^{U}}=0.7 \eta_{1}-\epsilon_{1}=0 \\
\frac{\partial L}{\partial b_{2}^{L}}=0.2 \eta_{2}-\phi_{2}=0 \\
\frac{\partial L}{\partial b_{2}^{U}}=0.8 \eta_{2}-\epsilon_{2}=0 \\
\frac{\partial L}{\partial D_{1}^{-}}=3.067+\xi_{1}+v_{1}-\zeta_{1}=0 \\
\frac{\partial L}{\partial D_{1}^{+}}=-\xi_{1}-\pi_{1}=0 \\
=0.839+\xi_{2}+v_{2}-\zeta_{2}=0 \\
\end{gathered}
$$




$$
\begin{aligned}
& \frac{\partial L}{\partial D_{2}^{+}}=-\xi_{2}-\pi_{2}=0, \\
& v_{1}\left[-x_{11}-3 x_{12}-x_{21}-2 x_{22}+D_{1}^{-}-2\right]=0 \text {, i.e., } v_{1}=0 \text {, } \\
& v_{2}\left[-x_{11}-2 x_{12}-3 x_{21}-x_{22}+D_{2}^{-}-4\right]=0 \text {, i.e., } v_{2}=0 \text {, } \\
& \tau_{1}\left[x_{11}+x_{12}-0.2 a_{1}^{L}-0.8 a_{1}^{U}\right]=0 \text {, i.e., } \tau_{1}=0, \\
& \tau_{2}\left[x_{21}+x_{22}-0.4 a_{2}^{L}-0.6 a_{2}^{U}\right]=0 \text {, i.e., } \tau_{2} \geq 0, \\
& \eta_{1}\left[-x_{11}-x_{21}+0.3 b_{1}^{L}+0.7 b_{1}^{U}\right]=0 \text {, i.e., } \eta_{1}=0, \\
& \eta_{2}\left[-x_{12}-x_{22}+0.2 b_{2}^{L}+0.8 b_{2}^{U}\right]=0 \text {, i.e., } \eta_{2}=0, \\
& \varphi_{1}\left[-x_{11}\right]=0 \text {, i.e., } \varphi_{1} \geq 0 \text {, } \\
& \varphi_{2}\left[-x_{12}\right]=0 \text {, i.e., } \varphi_{2}=0 \text {, } \\
& \varphi_{3}\left[-x_{21}\right]=0 \text {, i.e., } \varphi_{3}=0 \text {, } \\
& \varphi_{4}\left[-x_{22}\right]=0 \text {, i.e., } \varphi_{4}=0 \text {, } \\
& \psi_{1}\left[-a_{1}^{L}\right]=0 \text {, i.e., } \psi_{1}=0 \text {, } \\
& \psi_{2}\left[-a_{2}^{L}\right]=0 \text {, i.e., } \psi_{2}=0 \text {, } \\
& \phi_{1}\left[-b_{1}^{L}\right]=0 \text {, i.e., } \phi_{1}=0 \text {, } \\
& \phi_{2}\left[-b_{2}^{L}\right]=0 \text {, i.e., } \phi_{2}=0 \text {, } \\
& \omega_{1}\left[-a_{1}^{U}\right]=0 \text {, i.e., } \omega_{1}=0 \text {, } \\
& \omega_{2}\left[-a_{2}^{U}\right]=0 \text {, i.e., } \omega_{2}=0 \text {, } \\
& \epsilon_{1}\left[-b_{1}^{U}\right]=0 \text {, i.e., } \epsilon_{1}=0 \text {, } \\
& \epsilon_{2}\left[-b_{2}^{U}\right]=0 \text {, i.e., } \epsilon_{2}=0 \text {, } \\
& \zeta_{1}\left[-D_{1}^{-}\right]=0 \text {, i.e., } \zeta_{1} \geq 0, \\
& \zeta_{2}\left[-D_{2}^{-}\right]=0 \text {, i.e., } \zeta_{2}=0 \text {, } \\
& \pi_{1}\left[-D_{1}^{+}\right]=0 \text {, i.e., } \pi_{1} \geq 0 \text {, } \\
& \pi_{2}\left[-D_{2}^{+}\right]=0 \text {, i.e., } \pi_{2} \geq 0 \text {, } \\
& -x_{11}-3 x_{12}-x_{21}-2 x_{22}+D_{1}^{-} \leq 2 \text {, } \\
& -x_{11}-2 x_{12}-3 x_{21}-x_{22}+D_{2}^{-} \leq 4 \text {, } \\
& x_{11}+x_{12} \leq 0.2 a_{1}^{L}+0.8 a_{1}^{U}, \\
& x_{21}+x_{22} \leq 0.4 a_{2}^{L}+0.6 a_{2}^{U}, \\
& x_{11}+x_{21} \geq 0.3 b_{1}^{L}+0.7 b_{1}^{U}, \\
& x_{12}+x_{22} \geq 0.2 b_{2}^{L}+0.8 b_{2}^{U},
\end{aligned}
$$

$x_{11}, x_{12}, x_{21}, x_{22}, a_{1}^{L}, a_{1}^{U}, a_{2}^{L}, a_{2}^{U}, b_{1}^{L}, b_{1}^{U}, b_{2}^{L}, b_{2}^{U}, D_{1}^{-}, D_{1}^{+}, D_{2}^{-}, D_{2}^{+} \geq 0 ; \vartheta \in R$

Solving the above system of Equation. we get: $v_{1}=v_{2}=\tau_{1}=\tau_{2}=\eta_{1}=\eta_{2}=\varphi_{2}=$ $\varphi_{3}=\varphi_{4}=\psi_{1}=\psi_{2}=\phi_{1}=\phi_{2}=\omega_{1}=\omega_{2}=\epsilon_{1}=\epsilon_{2}=\zeta_{2}=0$, and $\varphi_{1}, \zeta_{1}, \pi_{1}, \pi_{2} \geq 0$. 
Also, $\xi_{2}=-\pi_{2}=-0.839, \xi_{1}=-\pi_{1}$. The above system of Equation is reduced to the following:

$$
\begin{gathered}
(-8.518+3.067 \vartheta) \xi_{1}-2.863 \xi_{2}-\varphi_{1}=0, \\
(-19.42+3.067 \vartheta) \xi_{1}+(-6.564+0.839 \vartheta) \xi_{2}=0, \\
(0.682+6.133 \vartheta) \xi_{1}+(-10.266+1.677 \vartheta) \xi_{2}=0, \\
1.364 \xi_{1}+(-0.347+0.839 \vartheta) \xi_{2}=0,
\end{gathered}
$$

Therefore, the SSFK for the parametric IF-MOFTP is given by:

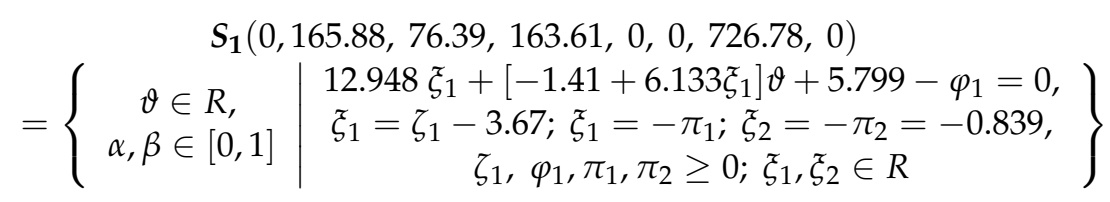

After applying the KKT optimality conditions we obtain a large system of algebraic equations. By reducing and solving the algebraic system of equations the SSFK is obtained. The SSFK introduces the values and relations between different parameters which generate the same solution of the PIF-MOFTP as indicated by set $S_{1}$. To test the obtained results of the SSFK, different values of $\alpha, \beta \in[0,1]$ will be taken and the solution will remain the same.

\section{Conclusions}

The SSFK for the PIF-MOFTP was investigated in this study. Also, we characterized definitions of the set of feasible parameters and the solvability set for PIF-MOFTP. First, the concept of $(\alpha, \beta)$-cut methodology was applied to get the parametric model. Moreover, the FGP approach was applied to find a $(\alpha, \beta)$-Pareto optimal solution for PIF-MOFTP which has not been published in the literature to date. To obtain the SSFK for the novel model of PIF-MOFTP, the KKT necessary optimality conditions are applied. After applying the KKT optimality conditions, we obtained a large system of algebraic equations. By reducing and solving the algebraic system of equations, the SSFK was obtained. A detailed procedure that determines the SSFK for the PIF-MOFTP was exhibited. A numerical example was given to ensure the applicability and efficiency of the proposed PIF-MOFTP.

The major limitation of the proposed PIF-MOFTP is that a specific $(\alpha, \beta)$-level is adopted in the proposed methods to represent the confidence level on DMs' subjective uncertainty to specify parameter values in the PIF-MOFTP. For simplification, the $(\alpha, \beta)$ level for all parameters of the supply and demand in the solution process are assumed to be the same. However, these may be limitations in practical applications. The determination of $(\alpha, \beta)$-levels for various $\mathrm{DMs}^{\prime}$ subjective uncertainties could be different in the real world due to DMs' different consideration of the real transportation data. Thus, this will be addressed in future studies.

Several remaining areas of research in the topic of parametric MOFTP include the following:

1. The parametric study of multi-choice MOTP should be addressed.

2. Real-world PIF-MOFTP is a vital field in the future research.

3. Rough parametric MOFTP is a vital topic to be investigated.

Author Contributions: Conceptualization, M.A.E.S., M.A.E.-S. and F.A.F.; Methodology, M.A.E.S., F.A.F. and M.A.E.; Investigation, M.A.E.S., M.A.E.-S., A.F.F. and M.A.E.; writing-review and editing, M.A.E.S., M.A.E.-S., A.F.F., M.A.E. and F.A.F. All authors have read and agreed to the published version of the manuscript.

Funding: This research received no external funding.

Institutional Review Board Statement: Not applicable. 
Informed Consent Statement: Not applicable.

Data Availability Statement: Not applicable.

Conflicts of Interest: The authors declare no conflict of interest.

\section{References}

1. Ammar, E.E.; Youness, E.A. Study on multi-objective transportation problem with fuzzy numbers. Appl. Math. Comput. 2005, 166, 241-253.

2. Bit, A.K.; Biswal, M.P.; Alam, S.S. Fuzzy programming approach to multi-criteria decision-making transportation problem. Fuzzy Sets Syst. 1992, 50, 135-141. [CrossRef]

3. Cetin, N.; Tiryaki, F. A Fuzzy Approach Using Generalized Dinkelbach's Algorithm for Multiobjective Linear Fractional Transportation Problem. Math. Probl. Eng. 2014, 2014, 702319. [CrossRef]

4. Chanas, S.; Kuchta, D. A concept of the optimal solution of the transportation problem with fuzzy cost coefficients. Fuzzy Sets Syst. 1996, 82, 299-305. [CrossRef]

5. Charnes, A.; Cooper, W.W. The steppingstone method for explaining linear programming calculation in transportation problem. Manag. Sci. 1954, 1, 49-69. [CrossRef]

6. Diaz, J.A. Finding a complete description of all efficient solutions to a multi-objective transportation problem. Ekon.-Mat. Obz. 1979, 15, 62-73.

7. El Sayed, M.; Abo-Sinna, M.A. A novel Approach for Fully Intuitionistic Fuzzy Multi-Objective Fractional Transportation Problem. Alex. Eng. J. 2020, 60, 1447-1463. [CrossRef]

8. Beaula, T.; Priyadharsini, M. A new algorithm for finding a fuzzy optimal solution for intuitionistic fuzzy transportation problems. Int. J. Appl. Fuzzy Sets Artif. Intell. 2015, 5, 183-192.

9. Arya, R.; Singh, P.; Kumari, S.; Obaidat, M.S. An approach for solving fully fuzzy multi-objective linear fractional optimization problems. Soft Comput. 2020, 24, 9105-9119. [CrossRef]

10. Ebrahimnejad, A.; Verdegay, J.L. A new approach for solving fully intuitionistic fuzzy transportation problems. Fuzzy Optim. Decis. Mak. 2017, 17, 447-474. [CrossRef]

11. Liou, J.J.; Hsu, C.-C.; Chen, Y.-S. Improving transportation service quality based on information fusion. Transp. Res. Part A Policy Pract. 2014, 67, 225-239. [CrossRef]

12. Ergun, O.; Kuyzu, G.; Savelsbergh, M. Reducing truckload transportation through collaboration. Transp. Sci. 2007, 41, 206-221. [CrossRef]

13. Sheu, J.B.; Chen, Y.J. Transportation and economics of scale in recycling low-value materials. Transp. Res. Part B Methodol. 2014, 65, 65-76. [CrossRef]

14. Roy, S.K.; Maity, G.; Weber, G.-W. Multi-objective two-stage grey transportation problem using utility function with goals. Cent. Eur. J. Oper. Res. 2016, 25, 417-439. [CrossRef]

15. Roy, S.K.; Maity, G.; Weber, G.W.; Gök, S.Z.A. Conic scalarization approach to solve multi-choice multi-objective transportation problem with interval goal. Ann. Oper. Res. 2017, 253, 599-620. [CrossRef]

16. Roy, S.K.; Mahapatra, D.R. Multi-objective interval valued transportation probabilistic problem involving lognormal. Int. J. Math. Sci. Comput. 2011, 1, 14-21.

17. Roy, S.K. Multi-choice stochastic transportation problem involving Weibull distribution. Int. J. Oper. Res. 2014, 21, 38. [CrossRef]

18. Maity, G.; Roy, S.K.; Verdegay, J.L. Multi-objective transportation problem with cost reliability under uncertain environment. Int. J. Comput. Intell. Syst. 2016, 9, 839-849. [CrossRef]

19. Maity, G.; Roy, S.K. Solving a multi-objective transportation problem with nonlinear cost and multi-choice demand. Int. J. Manag. Sci. Eng. Manag. 2014, 11, 62-70. [CrossRef]

20. Atanassov, K.T. Intuitionistic fuzzy sets. Fuzzy Sets Syst. 1986, 20, 87-96. [CrossRef]

21. Gong, Z.; Zhangc, N.; Chiclanad, F. The optimization ordering model for intuitionistic fuzzy preference relations with utility functions. Knowl.-Based Syst. 2018, 162, 174-184. [CrossRef]

22. Jana, B.; Roy, T.K. Multi-objective intuitionistic fuzzy linear programming and its application in transportation model. Notes Intuit. Fuzzy Sets 2007, 13, 34-51.

23. Verma, R.; Biswal, M.; Biswas, A. Fuzzy programming technique to solve multi-objective transportation problems with some non-linear membership functions. Fuzzy Sets Syst. 1997, 91, 37-43. [CrossRef]

24. Gourav, G.; Kumari, A. An efficient method for solving intuitionistic fuzzy transportation problem of type-2. Int. J. Appl. Comput. Math. 2017, 3, 3795-3804.

25. Gupta, A.; Kumar, A. A new method for solving linear multi-objective transportation problems with fuzzy parameters. Appl. Math. Model. 2012, 36, 1421-1430. [CrossRef]

26. Maity, G.; Roy, S.K. Solving multi-choice multi-objective transportation problem: A utility function approach. J. Uncertain. Anal. Appl. 2014, 2, 11. [CrossRef]

27. Mahajan, S.; Gupta, S.K. On fully intuitionistic fuzzy multiobjective transportation problems using different membership functions. Ann. Oper. Res. 2019, 296, 211-241. [CrossRef] 
28. El Sayed, M.; Farahat, F. Study of Achievement Stability Set for Parametric Linear FGP Problems. Ain Shams Eng. J. 2020, 11, 1345-1353. [CrossRef]

29. Veeramani, C.; Edalatpanah, S.A.; Sharanya, S. Solving the Multiobjective Fractional Transportation Problem through the Neutrosophic Goal Programming Approach. Discret. Dyn. Nat. Soc. 2021, 2021, 7308042. [CrossRef]

30. Pramanik, S.; Banerjee, D. Multi-objective chance constrained capacitated transportation problem based on fuzzy goal programming. Int. J. Comput. Appl. 2012, 44, 42-46. [CrossRef]

31. Edalatpanah, S.A. A nonlinear approach for neutrosophic linear programming. J. Appl. Res. Ind. Eng. 2019, 6, 367-373. [CrossRef]

32. Rizk-Allah, R.M.; Hassanien, A.E.; Elhoseny, M. A multi-objective transportation model under neutrosophic environment. Comput. Electr. Eng. 2018, 69, 705-719. [CrossRef]

33. Veeramani, C.; Sumathi, M. Fuzzy Mathematical Programming approach for Solving Fuzzy Linear Fractional Programming Problem. RAIRO-Oper. Res. 2014, 48, 109-122. [CrossRef]

34. Emam, O.E. A parametric study on multi-objective integer quadratic programming problems under uncertainty. Gen. Math. Notes 2011, 6, 49-60.

35. Osman, M.S. Qualitative analysis of basic notions in parametric convex programming, (parameters in the objective function). Apl. Mat. 1977, 22, 333-348. [CrossRef]

36. Osman, M.S.; Emam, O.E.; El Sayed, M.A. On Parametric Multi-level Multi-objective Fractional Programming Problems with Fuzziness in the Constraints. Br. J. Math. Comput. Sci. 2016, 18, 1-19. [CrossRef] [PubMed]

37. Hsien-Chung, W. The Karush-Kuhn-Tucker optimality conditions for multi-objective programming problems with fuzzy-valued objective functions. Fuzzy Optim. Decis. Mak. 2009, 8, 1-28.

38. Saad, O.M. On stability of proper efficient solutions in multi-objective fractional programming problems under fuzziness. Math. Comput. Model. 2007, 45, 221-231. [CrossRef]

39. Saad, O.M.; Hughes, J.B. Bicriterion integer linear fractional programs with parameters in the objective functions. J. Inf. Optim. Sci. 1998, 19, 97-108. [CrossRef]

40. Saad, O.M.; Elshafei, M.M.; Sleem, M.M. On some stability notions for fuzzy three-level fractional programming problem. Math. Sci. Lett. 2021, 10, 23-34.

41. Mahapatra, G.S.; Roy, T.K. Intuitionistic Fuzzy Number and Its Arithmetic Operation with Application on System Failure. J. Uncertain Syst. 2013, 7, 92-107.

42. Mahmoodirad, A.; Allahviranloo, T.; Niroomand, S. A new effective solution method for fully intuitionistic fuzzy transportation problem. Soft Comput. 2019, 23, 4521-4530. [CrossRef]

43. Roy, S.K.; Ebrahimnejad, A.; Verdegay, J.L.; Das, S. New approach for solving intuitionistic fuzzy multi-objective transportation problem. Sadhana 2018, 43, 3. [CrossRef]

44. Mohamed, R.H. The relationship between goal programming and fuzzy programming. Fuzzy Sets Syst. 1997, 89, 215-222. [CrossRef]

45. Pal, B.B.; Moitra, B.N.; Maulik, U. A goal programming procedure for fuzzy multi-objective linear fractional programming problem. Fuzzy Sets Syst. 2003, 139, 395-405. [CrossRef]

46. Zangiabadi, M.; Maleki, H.R. Fuzzy goal programming technique to solve multi-objective transportation problems with some non-linear membership functions. Iran. J. Fuzzy Syst. 2013, 10, 61-74.

47. Osman, M.; Emam, O.; El Sayed, M.A. Interactive Approach for Multi-Level Multi-Objective Fractional Programming Problems with Fuzzy Parameters. Beni-Suef Univ. J. Basic Appl. Sci. 2018, 7, 139-149. [CrossRef] 